\title{
Enteric Fever in Children Below 2 y - Need for an Effective Conjugate Vaccine?
}

\author{
Vidya Krishna • Padmasani Venkat Ramanan • \\ Priyadharshini Rajendran
}

Received: 31 August 2012 / Accepted: 8 August 2013 /Published online: 14 September 2013

(C) Dr. K C Chaudhuri Foundation 2013

To the Editor: The occurrence of enteric fever in children less than $2 \mathrm{y}$ is significant $[1,2]$ but not yet quantified. The vaccines currently available, target age group above $2 \mathrm{y}$ and whether there is a need for effective vaccines in children below $2 \mathrm{y}$ is a question that needs to be addressed.

We conducted a retrospective study of case records of children below 2 y, admitted between January 2009 and June 2012 with blood culture growth of S.typhi/S. paratyphi.

During this period, 48 children under 18 y of age were admitted with culture positive enteric fever of which 14 (29\%) were under $2 \mathrm{y}$, of which 7 (50\%) were under $1 \mathrm{y}$. Febrile gastroenteritis was the commonest presentation. Hepatomegaly was seen in $14.3 \%$ but none had icterus, rash or splenomegaly. Two children under 3 mo of age had meningitis.

Though total counts were normal in $78.6 \%$, absolute eosinopenia was universal. Two of the 14 blood cultures grew S. paratyphiand the rest, S. typhi. Most of the isolates $(92.9 \%)$ were resistant to Nalidixic acid. In both the infants with meningitis, CSF cell count and biochemistry were suggestive of typical bacterial meningitis but cultures were sterile. Blood culture in one of the two was positive for S. typhi and in the other for $S$. paratyphi.

The two children with meningitis were given I/V antibiotics for $4 \mathrm{wk}$. All others were treated with I/V Ceftriaxone or Cefotaxime till fever defervesence. Oral antibiotics were given at discharge to complete a 14-d regime. No deaths or relapses occurred.
This study draws attention to the problem of enteric fever in children under $2 \mathrm{y}$ of age. Reduction in incidence by improving water quality and sanitation is not immediately feasible in the developing countries. For short to intermediate term, vaccination is a control strategy recommended by WHO [3].

Large-scale epidemiological studies to assess burden of disease in children less than $2 \mathrm{y}$ and field trials for the safety and efficacy of the available Vi-rEPA and Vi-TT conjugate vaccines are necessary $[4,5]$.

\section{References}

1. Owais A, Sultana S, Zaman U, Rizvi A, Zaidi AK. Incidence of typhoid bacteremia in infants and young children in southern coastal Pakistan. Pediatr Infect Dis J. 2010;29:1035-9.

2. Verma M, Parashar Y, Singh A, Kamoji R. Current pattern of enteric fever: A prospective clinical and microbiological study. J Indian Med Assoc. 2007; 105: 582,584,586.

3. Typhoid vaccines: WHO position paper. In: Weekly epidemiological record. No.6, 2008, 83, 49-60. http://www.who.int/wer/2008/wer8306. pdf. Accessed 6th Jul 2013.

4. Thiem VD, Lin FY, Canhdo G, Son NH, Anh DD, Mao ND, et al. The Vi conjugate typhoid vaccine is safe, elicits protective levels of IgG anti- Vi, and is compatible with routine infant vaccines. Clin Vaccine Immunol. 2011;18:730-5.

5. Mathew JL. Conjugate typhoid vaccine(s) in the Indian context. Indian Pediatr. 2009;46:182-4.

\footnotetext{
V. Krishna $\cdot$ P. Rajendran

Department of Pediatrics, Sri Ramachandra Medical

College and Research Institute, Porur, Chennai, India

P. V. Ramanan $(\bowtie)$

Department of Pediatrics, Sri Ramachandra Medical College and

Research Institute, Porur, Chennai 600116, Tamil Nadu, India

e-mail: padmasani2001@yahoo.com
} 